\title{
Failure to access prescribed pharmaceuticals by older patients with chronic conditions
}

\author{
Ian McRae ${ }^{1,4} \mathrm{PhD}$, Research Fellow \\ Kees van $\mathrm{Gool}^{2} \mathrm{PhD}$, Associate Professor \\ Jane Hall ${ }^{2} \mathrm{PhD}$, Professor \\ Laurann Yen ${ }^{3}$ MPsych, Senior Fellow \\ Michael Wright ${ }^{2}$ MBBS, MSc, Research Fellow \\ ${ }^{1}$ Centre for Research on Ageing, Health and Wellbeing, Research School of Population Health, \\ The Australian National University, Florey, Building 54, Mills Road, Canberra, ACT 0200, Australia. \\ ${ }^{2}$ Centre for Health Economics Research and Evaluation, University of Technology Sydney, Level 2, \\ Building 5 Block D, 1-59 Quay Street, Haymarket, NSW 2000, Australia. Email: kees.vangool@uts.edu.au; \\ jane.hall@uts.edu.au; michael.wright@uts.edu.au \\ ${ }^{3}$ Department of Health Services Research and Policy Research, School of Population Health, The Australian \\ National University, 62A Mills Road, Canberra, ACT 0200, Australia. Email: laurann.yen@anu.edu.au \\ ${ }^{4}$ Corresponding author. Email: ian.s.mcrae@anu.edu.au
}

\begin{abstract}
Objective. Medication adherence is a significant public health concern. Australian studies of statins show patients facing the highest copayments are the least likely to be adherent. This study examined whether the association identified between adherence and costs for statins also applies to a wider group of medications prescribed for Australian patients with chronic conditions.

Methods. Data from 267086 participants in the Sax Institute's 45 and Up Study linked to data from the Pharmaceutical Benefits Scheme (PBS) provided by the Department of Human Services were used. Patients using angiotensin II receptor blockers, angiotensin-converting enzyme inhibitors, glitazones and bisphosphonates were identified and classified according to concessional status and whether they had access to the PBS 'safety net'. Data were analysed using mainly descriptive methods to investigate the association of adherence with cost and other selected covariates.

Results. Across medications, the group facing the highest copayment was least adherent. Speaking a language other than English at home and facing high levels of psychological distress were also associated with lower levels of adherence.

Conclusions. As for statins, the main financial determinant of adherence is cost in the form of prescribed copayments, suggesting that this may apply across many medications.

What is known about the topic? Previous studies have shown patients' concern about the costs of pharmaceuticals, and more detailed studies of statins show that the lowest adherence relates to patients facing the highest copayments.

What does this paper add? This paper provides support for the contention that the results found for statins broadly apply across more medications used by people with chronic conditions.

What are the implications for practitioners? Although practitioners cannot affect legislated copayments, they can consider the costs of options for medications for patients with chronic conditions, especially those general patients who have not reached the safety net, and they can be aware that patients from homes where English is not spoken and patients with high levels of psychological distress are also likely to have low adherence without intervention.
\end{abstract}

Received 16 July 2018, accepted 30 November 2018, published online 4 March 2019

\section{Introduction}

Medication adherence is a major concern for the health of any community $^{1}$ and numerous studies have identified financial, social, psychological and other barriers to adherence. ${ }^{1-4}$ Despite having a comprehensive safety net in the Pharmaceutical
Benefits Scheme (PBS), previous studies indicate that many Australian patients do not purchase prescribed medications for financial reasons. ${ }^{5-8}$

Australian studies ${ }^{8-10}$ have addressed factors associated with adherence in the use of statins, in particular showing that statin 
adherence was strongly related to the level of financial support to which patients were entitled. The purpose of this study was to extend these analyses to further medications to explore whether the results hold more widely.

\section{Australian institutional arrangements for pharmaceuticals}

Most prescriptions in Australia are filled privately and supported by the Australian Government's PBS. The PBS subsidises approved prescription medicines with fixed patient copayments, which are lower for patients holding government issued concession cards (more than one in four Australians ${ }^{11}$ ). Copayments are removed for concessional patients and reduced for non-concessional ('general') patients once a 'safety net threshold' of total annual copayments for a family is reached. This threshold is calculated each calendar year, with families paying the higher copayments unless they exceed the threshold for the relevant year. Table 1 shows the copayments and thresholds for 2008 (in the middle of the time period we analysed). We denote these copayment categories as concessional non-safety net (CNSN), concessional safety net (CSN), general non-safety net (GNSN) and general safety net (GSN).

Although few medications are priced below the concessional copayment, many are priced below the general copayment. General patients purchasing these medications who have not reached the safety net threshold pay a price determined by the pharmacist. Because there was no PBS contribution, these payments were not recorded in the PBS system during the period under investigation.

\section{Methods}

\section{Data sources}

The data source was the Sax Institute's 45 and Up Study, ${ }^{12}$ which is based in the population of the state of New South Wales (NSW), Australia. Prospective participants were randomly sampled from the Department of Human Services (formerly Medicare Australia) enrolment database, which provides nearcomplete coverage of the population. People aged $\geq 80$ years and residents of rural and remote areas were oversampled. In all, 267086 participants ( $\sim 11 \%$ of the NSW population and $\sim 18 \%$ of those invited) joined the 45 and Up Study by completing a baseline questionnaire (from 2006 to 2009) and giving signed consent for follow-up and linkage of their information to routine health databases.

The present study linked 45 and Up survey data with PBS data for the period 2005-11. Data linkage was completed by the Sax Institute using a unique identifier that was provided to the Department of Human Services.

PBS data cover all prescriptions funded under the PBS, and includes costs of each prescription, number of tablets or capsules, whether patients are general or concessional, and whether patients have reached a safety net. Self-reported data from the survey included a range of relevant social and demographic information.

\section{Medications assessed}

The medication classes included in the study were selected because they are typically long-term medications used to manage chronic conditions, have no perceivable immediate benefit for patients, are of sufficient price to be included in PBS data and are used by relatively large populations. It was also essential that the period that a particular prescription should cover was known, so medications that were used 'as needed' rather than a fixed number per day could not be included. For comparative purposes results are also reported for statins, some of which were reported previously ${ }^{8}$ and some of which are reported here for the first time.

The four medication classes were angiotensin II receptor blockers (ARBs), angiotensin-converting enzyme (ACE) inhibitors (both treatments for hypertension with similar adherence rates ${ }^{13,14}$ ), glitazones, used in the treatment of diabetes, and bisphosphonates, used in the treatment of osteoporosis. Glitazones (rosiglitazone and pioglitazone) are used to manage Type 2 diabetes when first-line therapy is inadequate or not tolerated. Adherence levels for glitazones are expected to be below those of antihypertensives, ${ }^{15}$ with those for bisphosphonates, which are complex to take, ${ }^{16,17}$ lower still (bisphosphonates must be taken on an empty stomach with no food for a period and patients must stay upright for half an hour after taking them)

Although glitazones and bisphosphonates were almost all priced above the general copayment level in the study period and only $5.4 \%$ of statin prescriptions covered fell below general copayment level, this is not the case for the blood pressure medications, with $89.0 \%$ of ARBs and $91.9 \%$ of ACE inhibitors in the categories other than GNSN being below the general copayment. Despite this, the blood pressure medications were included in this study because there are 147000 ARB prescriptions and 103000 ACE inhibitor prescriptions in the GNSN categories. However, care must be taken in addressing questions about these patients because they relate only to the more expensive formulations.

\section{Measure of adherence}

The ratio of the number of days notionally covered by a quantity of medication to the number of days actually covered is the medication possession ratio (MPR). ${ }^{9}$ For medications taken at one per day, the MPR is the quantity purchased divided by the number of days covered, assumed to be the time between purchases. For medications with different usage patterns the quantity must be converted to 'days covered' before calculating the MPR. We used the common threshold of an MPR $>80 \%$ to define adherence as a dichotomous variable. ${ }^{9}$

Adherence to medications can be considered within periods of initiation, implementation and discontinuation. ${ }^{1}$ In this study,

Table 1. Per prescription copayments and annual safety net thresholds for the Pharmaceutical Benefits Scheme, 2008 Data from Australian Government, Department of Health (http://www.pbs.gov.au/info/healthpro/explanatory-notes/front/fee, accessed 30 January 2019)

\begin{tabular}{lcc}
\hline & Copayment (A\$) & Family safety net threshold (A\$) \\
\hline Concessional patients (pension or healthcare cards) & 5.00 & 290.00 \\
General patients (non-concessional) & 31.30 & 1141.80 \\
\hline
\end{tabular}


we are dealing with periods within a year, so we are primarily examining the implementation period. Adherence measures are generally lower for people who fail to initiate and those discontinuing $^{18}$ than for those in the implementation phase, so we expected higher estimates of adherence than those studies that included initiation and discontinuation periods.

\section{Categories}

To allocate person-years to categories (e.g. CSN), we allocated survey participants to concessional or general categories depending on which comprised the majority of all prescriptions purchased by a given person in the year. We then defined the group as 'safety net' if more than $20 \%$ of prescriptions for that person-year were under safety net arrangements, based on the view that $20 \%$ will affect behaviour. As reported previously, sensitivity analysis showed little difference between using $10 \%$ and $30 \%$ of prescriptions to define the safety net group. ${ }^{8}$

\section{Analysis}

Because the safety net system operates on an annual cycle, for each selected medication we examined annual adherence by copayment category. We further examined changes in adherence when patients moved between non-safety net and safety net categories within each year. Finally, for a set of selected covariates, we report the ratio of mean adherence for those in the 'active' group (e.g. holding private health insurance) to those in the 'inactive' group for each medication within each category, as well as the significance of the difference between the mean adherence in the 'active' and 'inactive' groups, determined using a standard $t$-test for comparison of means of two groups with unequal variances. The variables selected were language spoken at home, private health insurance, degree of psychological distress and regular smoking. These were the most significant in the previous statin modelling, ${ }^{8-10}$ and in modelling undertaken for the additional medications. For reasons of parsimony, these models are not reported here, but are available from the authors. Relative adherence rates are also reported for high and low levels of income (defined as above or below A $\$ 50000$ per annum) because this was a material consideration in relation to statins, as well as for the dosage frequency for bisphosphonates, which is a specific issue for these medications.

The Sax Institute's 45 and Up Study was approved by the University of NSW Human Research Ethics Committee. The project was approved by the NSW Population and Health Services Research Ethics Committee (Reference no. 2013/11/487).
Analysis was undertaken in Stata ver. 12.1 (StataCorp LP, College Station, TX, USA).

\section{Results}

\section{Sample structure}

The relevant number of people and prescriptions in the 45 and Up dataset overall and the number of persons and person-years used in the analysis following exclusion of implausible or unusable data are given in Table 2.

Person-years excluded were those that could not be analysed (e.g. all prescriptions on one day, values missing), where adherence measures were not meaningful (e.g. $<3$ or $>24$ prescriptions in a year), where average cost per individual 'pill' was more than A \$1.30, which is well above the maximum copayment for these medications, where the average quantity purchased per day covered was more than threefold standard usage and where there was a gap of $\geq 6$ months between purchases (including periods across years).

Each medication class includes formulations that are combined with other medications. For example, some ARBs are provided alone and some are provided in combination with other blood pressure management medications. For completeness we included both the combinations and the medications used alone. Like statins, ARBs and ACE inhibitors are mainly dispensed in packets of 28 to be taken one per day; small numbers are dispensed in other sizes, and these were excluded. Although most prescriptions for glitazones relate to packs of 28 pills (taken at one per day), $9.0 \%$ have 56 pills taken at two per day, and adjustment has been made for this usage rate, with packs of other sizes excluded. Bisphosphonates are available in daily, weekly, monthly and annual formulations. ${ }^{16}$ We excluded the annual version and converted the weekly and monthly to daily equivalents.

\section{Adherence patterns}

As indicated in Table 3, the average rate of adherence for the tested medications was closely related to the concessional and safety net categories, with similar patterns across medications. There are two consistent factors: the GNSN groups have the lowest adherence levels and the safety net categories have higher adherence than the comparative non-safety net categories.

Because our measure of adherence did not address discontinuation or failure to initiate (see above), ${ }^{1}$ and we excluded person-years with few prescriptions, the estimated levels of medication adherence are somewhat higher than those reported elsewhere, although there are wide ranges of estimates in the literature. ${ }^{9,14-16}$ A previous study using the same dataset but

Table 2. Sample structures for person-year data

$\mathrm{ACE}$, angiotensin-converting enzyme; ARBs, angiotensin receptor blockers

\begin{tabular}{|c|c|c|c|c|}
\hline Medication & $\begin{array}{l}\text { Total no. people claiming for this } \\
\text { medication over the period }\end{array}$ & $\begin{array}{c}\text { Total no. prescriptions } \\
\text { claimed }\end{array}$ & $\begin{array}{l}\text { No. people in } \\
\text { final sample }\end{array}$ & $\begin{array}{l}\text { No. person-years } \\
\text { in final sample }\end{array}$ \\
\hline Statins & 106808 & 4744153 & 94114 & 442344 \\
\hline ACE inhibitors & 60370 & 2251920 & 47978 & 195862 \\
\hline Glitazones & 3770 & 107978 & 3159 & 10067 \\
\hline Bisphosphonates & 19252 & 586170 & 15969 & 53716 \\
\hline
\end{tabular}


Table 3. Relationship between safety net categories and mean adherence

Data show mean percentage adherence in each category, with $95 \%$ confidence intervals in parentheses. ACE, angiotensin-converting enzyme; ARBs, angiotensin receptor blockers

\begin{tabular}{lccccc}
\hline Category & Statins $^{8}$ & ARBs & ACE inhibitors & Glitazones & Bisphosphonates \\
\hline Concessional safety net & $90.8(90.6-90.9)$ & $91.6(91.4-91.8)$ & $92.3(92.1-92.5)$ & $89.9(89.1-90.8)$ & $85.4(84.9-85.9)$ \\
Concessional non-safety net & $82.4(82.2-82.5)$ & $87.2(87.0-87.4)$ & $88.1(87.9-88.3)$ & $81.8(80.3-83.4)$ & $82.2(81.7-82.7)$ \\
General safety net & $86.0(85.7-86.4)$ & $93.8(93.5-94.2)$ & $93.5(93.1-93.9)$ & $89.5(88.0-90.9)$ & $82.6(81.2-84.0)$ \\
General non-safety net & $70.0(69.7-70.3)$ & $80.3(79.4-81.2)$ & $84.7(83.8-85.7)$ & $78.3(75.9-80.7)$ & $77.9(76.8-78.9)$ \\
Total & $82.6(82.5-82.7)$ & $89.1(89.0-89.2)$ & $90.0(89.8-90.1)$ & $86.6(86.0-87.3)$ & $83.0(82.7-83.3)$ \\
\hline
\end{tabular}

Table 4. Changes in adherence status between non-safety net and safety net categories ACE, angiotensin-converting enzyme; ARBs, angiotensin receptor blockers

\begin{tabular}{|c|c|c|c|c|c|c|c|}
\hline & \multicolumn{2}{|c|}{$\begin{array}{l}\text { People with no safety } \\
\text { net prescriptions }\end{array}$} & \multicolumn{2}{|c|}{$\begin{array}{l}\text { People adherent in non-safety net } \\
\text { period with safety net prescriptions }\end{array}$} & \multicolumn{2}{|c|}{$\begin{array}{l}\text { People not adherent in non- } \\
\text { safety net period with safety net } \\
\text { prescriptions }\end{array}$} & \multirow[t]{2}{*}{$\begin{array}{l}\text { Total no. } \\
\text { periods }^{\mathrm{A}}\end{array}$} \\
\hline & $\begin{array}{l}\text { No. } \\
\text { periods }^{A}\end{array}$ & $\begin{array}{l}\% \text { Adherent in non- } \\
\text { safety net period }\end{array}$ & No. periods ${ }^{\mathrm{A}}$ & $\begin{array}{l}\% \text { Adherent in } \\
\text { safety net period }\end{array}$ & $\begin{array}{c}\text { No. } \\
\text { periods }^{\mathrm{A}}\end{array}$ & $\begin{array}{l}\% \text { Adherent in } \\
\text { safety net period }\end{array}$ & \\
\hline \multicolumn{8}{|l|}{ Concessional } \\
\hline Statins & 162096 & 79.7 & 127133 & 87.9 & 11006 & 74.7 & 300235 \\
\hline ARBs & 110601 & 83.0 & 77433 & 88.4 & 10207 & 67.5 & 198241 \\
\hline ACE inhibitors & 97777 & 82.4 & 68164 & 88.9 & 10067 & 68.2 & 176008 \\
\hline Glitazones & 2453 & 83.4 & 4014 & 90.2 & 377 & 69.8 & 6844 \\
\hline Bisphosphonates & 24819 & 81.7 & 16901 & 88.3 & 2873 & 66.8 & 44593 \\
\hline \multicolumn{8}{|l|}{ General } \\
\hline Statins & 104223 & 69.2 & 29170 & 89.5 & 3689 & 79.4 & 137082 \\
\hline ARBs & 12946 & 73.8 & 3939 & 92.7 & 1347 & 75.6 & 18232 \\
\hline ACE inhibitors & 8348 & 75.1 & 3108 & 96.0 & 1044 & 75.9 & 12500 \\
\hline Glitazones & 1404 & 81.9 & 1322 & 91.6 & 137 & 70.1 & 2863 \\
\hline Bisphosphonates & 6831 & 78.9 & 2102 & 88.7 & 429 & 71.3 & 9362 \\
\hline
\end{tabular}

A‘Periods' are those parts of person-years (including whole person-years) with scripts covered by the safety net or not, as appropriate.

incorporating initiation and discontinuation estimated $80.1 \%$ adherence for concessional patients and $56.7 \%$ adherence for general patients for statins. ${ }^{9}$

As indicated in Table 4, most patients did not reach the safety nets. For both concessional and general patients, and for all medications, approximately $90 \%$ of those adherent before reaching the safety net remain adherent, whereas of those not adherent before reaching the safety net between $68 \%$ and $76 \%$ become adherent. Therefore, the high levels of adherence for safety net categories in Table 3 are due to the combination of patients adherent in the non-safety net period maintaining their adherence and patients non-adherent in the pre-safety net period becoming adherent when copayments are reduced.

\section{Covariates}

Table 5 provides the relative mean levels of adherence for high and low incomes, as well as for the set of covariates outlined in the Methods.

Consistent with findings for statins reported previously, ${ }^{8}$ in the case of blood pressure medications, among those in the general non-safety net category, those with a lower income have lower adherence and, among the concessional safety net category, people with a low income have higher adherence. All but one of these estimates are significant. However, patterns of adherence to glitazones and bisphosphonates were different and were mostly not significant.

Almost all comparisons for speaking a language other than English at home, for having higher levels of psychological distress and for current smoking showed lower levels of adherence for the 'active' group. These estimates are generally significant, with levels of significance declining with smaller samples. Conversely, private health insurance was associated with higher levels of adherence for the active group (i.e. those holding insurance), again with most estimates being significant and broadly lower levels of significance with smaller samples.

Although for bisphosphonates comparisons of daily and monthly dosages were not significant due to the relatively few patients with daily dosages, all ratios showed that a longer duration between doses was associated with higher adherence, and three of the four monthly-weekly comparisons were significant.

\section{Discussion}

The purpose of this study was to observe whether conclusions drawn about the role of costs in adherence to statins ${ }^{8}$ in Australia can be applied to other medications. For all the medications 
Table 5. Major covariates $(P<0.05$ was considered significant $)$

$\mathrm{ACE}$, angiotensin-converting enzyme; ARBs, angiotensin receptor blockers

\begin{tabular}{|c|c|c|c|c|c|c|c|c|}
\hline & \multicolumn{2}{|c|}{$\begin{array}{c}\text { Concessional } \\
\text { non-safety net }\end{array}$} & \multicolumn{2}{|c|}{ Concessional safety net } & \multicolumn{2}{|c|}{ General non-safety net } & \multicolumn{2}{|c|}{ General safety net } \\
\hline \multicolumn{9}{|l|}{ Income } \\
\hline $\mathrm{ARB}$ & 0.990 & $<0.05$ & 0.981 & $<0.001$ & 1.031 & $<0.05$ & 1.001 & NS \\
\hline ACE inhibitors & 0.991 & $<0.05$ & 0.983 & $<0.01$ & 1.017 & NS & 0.998 & NS \\
\hline Glitazones & 0.936 & NS & 0.953 & NS & 0.940 & NS & 0.974 & NS \\
\hline \multicolumn{9}{|l|}{ Private health insurance } \\
\hline Statins & 1.052 & $<0.001$ & 1.016 & $<0.001$ & 1.118 & $<0.001$ & 1.032 & $<0.001$ \\
\hline $\mathrm{ARB}$ & 1.023 & $<0.001$ & 1.004 & NS & 1.065 & $<0.001$ & 1.006 & NS \\
\hline ACE inhibitors & 1.031 & $<0.001$ & 1.005 & $<0.05$ & 1.051 & $<0.001$ & 0.994 & NS \\
\hline Glitazones & 1.058 & $<0.01$ & 0.994 & NS & 1.000 & NS & 1.077 & $<0.01$ \\
\hline Bisphosphonates & 1.031 & $<0.001$ & 1.010 & NS & 1.065 & $<0.01$ & 1.073 & $<0.05$ \\
\hline Glitazones & 0.863 & $<0.001$ & 0.948 & $<0.001$ & 0.938 & NS & 0.954 & NS \\
\hline Bisphosphonates & 0.964 & $<0.001$ & 1.004 & NS & 0.972 & NS & 0.963 & NS \\
\hline \multicolumn{9}{|c|}{ Kessler Psychological Distress Scale } \\
\hline Statins & 0.918 & $<0.001$ & 0.974 & $<0.001$ & 0.900 & $<0.001$ & 0.942 & $<0.001$ \\
\hline ARB & 0.937 & $<0.001$ & 0.979 & $<0.001$ & 0.940 & $<0.01$ & 0.977 & $<0.001$ \\
\hline ACE inhibitors & 0.938 & $<0.001$ & 0.972 & $<0.001$ & 0.975 & NS & 0.988 & NS \\
\hline Glitazones & 0.902 & $<0.001$ & 0.963 & $<0.01$ & 0.797 & $<0.001$ & 0.958 & NS \\
\hline Bisphosphonates & 0.916 & $<0.001$ & 0.938 & $<0.001$ & 0.993 & NS & 1.039 & NS \\
\hline \multicolumn{9}{|l|}{ Current regular smoking } \\
\hline Statins & 0.905 & $<0.001$ & 0.973 & $<0.001$ & 0.911 & $<0.001$ & 0.968 & $<0.01$ \\
\hline $\mathrm{ARB}$ & 0.956 & $<0.001$ & 0.995 & NS & 0.973 & NS & 0.986 & NS \\
\hline ACE inhibitors & 0.940 & $<0.001$ & 0.980 & $<0.001$ & 0.947 & $<0.05$ & 1.002 & NS \\
\hline
\end{tabular}

${ }^{\mathrm{A}}$ The ratios of mean adherence are for: (1) a high (i.e. above A $\$ 50000$ per year) versus low family income; (2) having versus not having private health insurance; (3) speaking versus not speaking a language other than English at home; (4) having greater than a 'low' level of distress versus a low level of distress, as measured by the Kessler Psychological Distress Scale; (5) currently smoking regularly versus not smoking regularly; and (6) for the frequency with which bisphosphonate medication is taken, weekly versus daily and monthly versus weekly.

tested in this study, the patients facing the highest out-of-pocket costs (the GNSN patients) had the lowest adherence levels. For both general and concessional patient groups, those on the safety net always had higher adherence levels. This result is driven, in part, by more than two-thirds of those not adherent before reaching the safety net becoming adherent after reaching it.

Although the results of this study fit the broad expectations of how people respond to changing prices, our ability to examine these issues at a household level is limited. Further research in this area is warranted, given that both financial constraints and safety net thresholds apply at a family level rather than an individual patient level. Future analysis should address family-level decision making, and whether there is prioritising in drug purchasing within a family. Unfortunately, the data used in this analysis do not define families, and so are not amenable to addressing this issue, and it would be necessary to obtain household-level data in order to explore this issue.
One factor that provided additional support for our views regarding the effects of costs on statin management ${ }^{8}$ was the role of income, because the GNSN patients with the lowest income had the lowest level of adherence. Although this pattern was broadly followed in the blood pressure medications, for glitazones and bisphosphonates the effect of income was mostly insignificant, with no clear patterns. These results may be due, in part, to the relatively small samples for glitazones and bisphosphonates, but also because these medications have greater barriers in the form of more specific indications (first-line diabetes measures failed or the patient has a fracture aligned with osteoporosis), and the doctor must obtain 'authority' from the Department of Human Services, generally by telephone, in order to prescribe them. Although these results do not detract from the conclusion that the GNSN patients have the poorest levels of adherence, they do suggest that for some medications the low-income segment of this category is little different to the 
higher-income patients. GNSN patients using blood pressure medications do not comprise a complete sample because some are prescribed below copayment formulations, meaning there is a risk that the behaviour of those in our sample may be different to that of patients prescribed the lower-cost formulations for whom we have no information. Although it is likely that adherence rates will be higher in the latter group because they face lower prices, and it is also likely that the effect of income would be similar in these two subgroups, there is no information on the excluded group to confirm this expectation.

As for previous studies, across all medications and categories, speaking a language other than English at home has negative associations with adherence, as does having 'more than low levels of distress' on the Kessler psychological distress scale. Adherence was generally associated with holding private health insurance, which may be a 'healthy adherer bias', 19 with those more attentive to their health both adhering to prescribed medicines and holding private health insurance, and was negatively associated with being a smoker, possibly the converse of the 'healthy adherer bias'. The private health insurance result could also be an effect of income, because people with a higher income are more likely to hold private health insurance. For concessional patients with a family income below $\mathrm{A} \$ 50000,47 \%$ held private health insurance, compared with $87 \%$ of those with higher incomes. For general patients, the respective proportions are $70 \%$ and $89 \%$. We also confirmed the well-known result ${ }^{16}$ that adherence to bisphosphonates is better when the medication must be taken less frequently.

Overall, the main results of our previous analysis, ${ }^{8}$ and hence the policy conclusions, apply. If adherence levels are to be improved, the place to start, noting the large differences in legislated copayments between categories of patients, is the GNSN group. Adherence across this set of medications could be improved by reducing out-of-pocket costs for the GNSN group in various ways, including simply reducing copayments, reducing the safety net threshold or broadening definitions of 'concessional'. One approach that avoids much of the funding going to people already adherent would be a stepped approach with two or three thresholds, and smaller copayment reductions. In addition, to reducing financial barriers, provision of medical advice in home languages for those not using English at home, better awareness by health providers of the need to support those in psychological distress with managing their medications and further advice to prescribers on the optimal forms of bisphosphonate would be valuable.

\section{Study limitations}

The main constraint on selecting medications is that prescriptions that cost less than the general copayment were not recorded on the PBS for the period under investigation. We included general patients for ARBs and ACE inhibitors where some of the scripts are priced below the general copayment because they have large samples, and their results prove to be broadly consistent with results from the other medications.

\section{Conclusions}

Using linked survey and administrative data, medication adherence can be explored with drugs used on a long-term basis for chronic conditions. In this study we compared four groups of medications with the previously studied statins, and consistently found that higher out-of-pocket costs per prescription were associated with poorer medication adherence. Although the number of medications studied remains limited, the consistency of the results suggests that they are likely to apply more widely.

In particular, for each of the target medications, the GNSN group, which faces the highest out-of-pocket costs, was the least adherent category. Because many patients are on multiple medications, the effects of cost on the therapeutic management of complex and chronic illness may be especially compromised. If the full benefits of these medications are to be available, consideration should be given to reducing the financial barriers for GNSN patients, potentially using a stepped safety net arrangement.

This study also provides results in relation to non-financial variables that are consistent with previous studies, and with clear implications for measures that would improve adherence to these medications, and hence their effectiveness and value to the community.

\section{Competing interests}

The authors have no competing interests to declare.

\section{Acknowledgements}

This research was completed using data collected through the 45 and Up Study (www.saxinstitute.org.au, accessed 10 July 2018). The 45 and Up Study is managed by the Sax Institute in collaboration with major partner Cancer Council NSW and other partners, namely the National Heart Foundation of Australia (NSW Division), NSW Ministry of Health, NSW Government Family and Community Services - Ageing, Carers and the Disability Council NSW, and the Australian Red Cross Blood Service. The authors thank the many thousands of people participating in the 45 and Up Study. The linked Medicare Benefits Schedule and Pharmaceutical Benefits Scheme data were supplied to the 45 and Up Study by the Commonwealth Department of Human Services. The research was funded by the Research in the Finance and Economics of Primary Health Care Centre of Research Excellence (ReFinE-PHC) under the Australian Primary Health Care Institute's (APHCRI) Centres of Research Excellence funding scheme, which is supported by a grant from the Australian Government Department of Health. The paper does not necessarily reflect the views of APHCRI or the Australian Government.

\section{References}

1 Vrijens B, De Geest S, Hughes DA, Przemyslaw K, Demonceau J, Ruppar T, Dobbels F, Fargher E, Morrison V, Lewek P, Matyjaszczyk M, Mshelia C, Clyne W, Aronson JK, Urquhart J. ABC Project Team. A new taxonomy for describing and defining adherence to medications. Br J Clin Pharmacol 2012; 73: 691-705. doi:10.1111/j.1365-2125. 2012.04167.x

2 Abbass I, Revere L, Mitchell J, Appari A. Medication nonadherence: the role of cost, community, and individual factors. Health Serv Res 2017; 52: 1511-33. doi:10.1111/1475-6773.12547

3 Kardas P, Lewek P, Matyjaszczyk M. Determinants of patient adherence: a review of systematic reviews. Front Pharmacol 2013; 4: 91.

4 Briesacher BA, Gurwitz JH, Soumerai SB. Patients at-risk for costrelated medication nonadherence: a review of the literature. J Gen Intern Med 2007; 22: 864-71. doi:10.1007/s11606-007-0180-x

5 Schoen C, Osborn R, Squires D, Doty MM, Pierson R, Applebaum S. How health insurance design affects access to care and costs, by income, 
in eleven countries. Health Aff (Millwood) 2010; 29: 2323-34. doi:10.1377/hlthaff.2010.0862

6 Menzies Centre for Health Policy. Survey of attitudes towards the Australian health system - part 2: financial stress and the Australian health system. 2008. Available at: https://ses.library.usyd.edu.au//bitstream/2123/19909/2/2008surveyfinancialsummary.pdf [verified 3 February 2019].

7 Australian Bureau of Statistics (ABS). 4839.0 - Patient experiences in Australia: summary of findings, 2015-16. 2016. Available at: http:// www.abs.gov.au/AUSSTATS/abs@.nsf/DetailsPage/4839.02015-16? OpenDocument [verified 18 December 2016].

8 McRae I, van Gool K, Hall J, Yen L. The role of cost on failure to access prescribed pharmaceuticals - the case of statins. Appl Health Econ Health Policy 2017; 15: 625-34.

9 Warren JR, Falster MO, Fox D, Jorm L. Factors influencing adherence in long-term use of statins. Pharmacoepidemiol Drug Saf 2013; 22: 1298-307. doi:10.1002/pds.3526

10 Warren JR, Falster MO, Tran B, Jorm L. Association of continuity of primary care and statin adherence. PLoS One 2015; 10: e0140008. doi:10.1371/journal.pone. 0140008

11 Department of Social Services. DSS payment demographic data. 2015. Available at: https://data.gov.au/dataset/ds-dga-cff2ae8a-55e4-47dba66d-e177fe0ac6a0/distribution/dist-dga-6b3fba11-2f71-4df9-963d-fc e31a7e59e3/details?q= [verified 30 January 2019].

1245 and Up Study Collaborators. Cohort profile: the 45 and up study. Int $J$ Epidemiol 2008; 37: 941-7. doi:10.1093/ije/dym184
13 Burnier M. Medication adherence and persistence as the cornerstone of effective antihypertensive therapy. Am J Hypertens 2006; 19: 1190-6. doi:10.1016/j.amjhyper.2006.04.006

14 Kronish IM, Woodward M, Sergie Z, Ogedegbe G, Falzon L, Mann DM. Meta-analysis: impact of drug class on adherence to antihypertensives. Circulation 2011; 123: 1611-21. doi:10.1161/CIRCULATIONAHA.110. 983874

15 Barner JC. Adherence to oral antidiabetic agents with pioglitazone and metformin: comparison of fixed-dose combination therapy with monotherapy and loose-dose combination therapy. Clin Ther 2011; 33: 1281-8. doi:10.1016/j.clinthera.2011.07.016

16 Rampakakis E, Sampalis JS. What can be done to maximise adherence of biphosphonates in patients with osteoporosis? Int J Clin Rheumtol 2012; 7: 361-4. [Editorial] doi:10.2217/ijr.12.41

17 Bell JS, Blacker N, Edwards S, Frank O, Alderman CP, Karan L, Husband A, Rowett D. Osteoporosis: pharmacological prevention and management in older people. Aust Fam Physician 2012; 41: 110-18.

18 Karter AJ, Parker MM, Solomon MD, Lyles CR, Adams AS, Moffet HH, Reed ME. Effect of out-of-pocket cost on medication initiation, adherence, and persistence among patients with yype 2 diabetes: The Diabetes Study of Northern California (DISTANCE). Health Serv Res 2018; 53(2): 1227-47. doi:10.1111/1475-6773.12700

19 Shrank WH, Patrick AR, Alan Brookhart M. Healthy user and related biases in observational studies of preventive interventions: a primer for physicians. J Gen Intern Med 2011; 26: 546-50. doi:10.1007/s11606-010-1609-1 